Pacific Journal of Mathematics

PRIMARY GROUPS WHOSE SUBGROUPS OF SMALLER 


\title{
PRIMARY GROUPS WHOSE SUBGROUPS OF SMALLER CARDINALITY ARE DIRECT SUMS OF CYCLIC GROUPS
}

\author{
PAUL HILL
}

Let $G$ denote a primary abelian group. The conjecture (partially supported by a theorem of Nunke) that there exists, for each infinite cardinal $m$, a group $G$ of cardinality $m$ that is not a direct sum of cyclic groups but has the property that each subgroup of $G$ having cardinality less than $m$ is a direct sum of cyclic groups is shown to be false. More specifically, it is shown that if a primary group has cardinality $\aleph_{\omega}$ and each subgroup of smaller cardinality is a direct sum of cyclic groups, then so is the group.

Further, we show that if $G$ is the union of a countable chain of pure subgroups, then $G$ is a direct sum of cyclic groups if and only if the subgroups in the given chain are direct sums of cyclic groups.

All groups considered here are primary abelian groups. For simplicity of terminology, we say that the group $G$ is an $m$-group if $G$ has cardinality $m$ and each subgroup of $G$ having cardinality less than $m$ is a direct sum of cyclic groups. The following problem, in one form of the other, has received considerable attention in recent years.

Problem. For which cardinals $m$ is evely $m$-group a direct sum of cyclic groups?

That every $m$-group is a direct sum of cyclic groups for a finite cardinal $m$ follows immediately from the well-known structure of finite abelian groups. Prüfer [8] discovered early two interesting examples of an $\aleph_{0}$-group that is not a direct sum of cyclic groups: one of his examples was divisible and the other was reduced. Each of the two examples, but especially the reduced one, is often called the Prüfer group. Recently, Nunke [7] has shown, for any nonnegative integer $n$, the existence of an $m$-group that is not a direct sum of cyclic groups with $m \geqq \boldsymbol{\aleph}_{n}$. It has been informally conjectured that for every infinite cardinal $m$ there exists an $m$-group that is not a direct sum of cyclic groups. However, the following theorem shows that this is not the case.

THEOREM 1. Let $G$ be an arbitrary primary abelian group. If 
$G$ is an $\aleph_{\omega}$-group, then $G$ is necessarily a direct sum of cyclic groups.

Proof. Let $G$ be a group of cardinality $\boldsymbol{W}_{\omega}$ such that each subgroup of $G$ having cardinality less than $\aleph_{\omega}$ is a direct sum of cyclic groups. Since any infinite subgroup can be imbedded in a pure subgroup of the same cardinality, it follows at once that $G$ is the union of an ascending sequence of pure subgroups $H_{n}$ of $G$ such that $|H|=\aleph_{n}$ for $0 \leqq n<\omega$. For each $n<\omega$, let

$$
H_{n}=\sum_{i \in I(n)}\left\{g_{i}\right\} \text {. }
$$

For simplicity of notation, let $\mu$ denote the smallest ordinal having cardinality $\boldsymbol{\aleph}_{\omega}$. We claim that there exist subgroups $A_{\alpha}$ of $G$, for $\alpha<\mu$, such that

(0) $A_{0}=0$.

(1) $A_{\alpha}$ is pure in $G$ for each $\alpha<\mu$.

(2) $\left\{A_{\alpha}, H_{n}\right\}$ is pure in $G$ for each $\alpha<\mu$ and each $n<\omega$.

(3) $A_{\alpha+1} \supseteqq A_{\alpha}$ for each $\alpha$ such that $\alpha+1<\mu$.

(4) $A_{\alpha+1} / A_{\alpha}$ is countable for each $\alpha$ such that $\alpha+1<\mu$.

(5) $A_{\alpha} \cap H_{n}=\sum_{i \in I(n, \alpha)}\left\{g_{i}\right\}$ for $\alpha<\mu$ and $n<\omega$, where $I(n, \alpha)$ is a subset of $I(n)$.

(6) $A_{\beta}=\mathrm{U}_{\alpha<\beta} A_{\alpha}$ if $\beta$ is a limit ordinal less than $\mu$.

(7) $G=\bigcup_{\alpha<\mu} A_{\alpha}$.

The proof of the existence of subgroups $A_{\alpha}, \alpha<\mu$, satisfying conditions (0) - (7) is, of course, by transfinite induction, and it employs the back-and-forth technique utilized in [1], [2], [3], [4], [5], [6] and a number of other recent papers. The reader who is well acquainted with these papers may need no further details concerning the existence of the subgroups $A_{\alpha}$ satisfying conditions (0) - (7); however, we shall include a brief outline of the proof.

Let $\gamma<\mu$, and suppose that we have chosen a subgroup $A_{\alpha}$ of $G$ for each $\alpha<\gamma$ such that conditions (0)-(6) hold when $\mu$ is replaced by $\gamma$. We wish to select $A_{\gamma}$ such that it is compatible with these conditions also. There are two cases.

Case 1. $\gamma$ is a limit ordinal. In this case, we let $A_{\gamma}=\mathrm{U}_{\alpha<r} A_{\alpha}$. Observe that $A_{\gamma}$ is pure in $G$ since $A_{\alpha}$ is for each $\alpha<\gamma$. In the same way, $\left\{A_{\gamma}, H_{n}\right\}$ is pure $G$. Furthermore, we have honored condition (6) in the definition of $A_{r}$. If we set $I(n, \gamma)=\mathrm{U}_{\alpha<r} I(n, \alpha)$ for each $n$, then it is easy to prove that $A_{r} \cap H_{n}=\sum_{i \in I(n, r)}\left\{g_{i}\right\}$. Hence all of the conditions (0) - (6) continue to hold if we pass from $\alpha<\gamma$ to $\alpha \leqq \gamma$.

Case 2. $\gamma-1$ exists. We want to show that there exists a 
subgroup $A_{\gamma}$ of $G$ such that $A_{\gamma}$ is a countable extension of $A_{\gamma-1}$ and such that

(i) $A_{r}$ is pure in $G$.

(ii) $\left\{A_{r}, H_{n}\right\}$ is pure in $G$ for each $n<\omega$.

(v) $A_{\gamma} \cap H_{n}=\sum_{i \in I(n, r)}\left\{g_{i}\right\}$ for each $n$, where $I(n, \gamma)$ is a subset of $I(n)$.

Let $B$ be any subgroup of $G$ containing $A_{\gamma-1}$. If $B / A_{\gamma-1}$ is countable, there exists, according to Theorem 1 in [3], $C \supseteqq B$ with $|C| A_{r-1} \mid \leqq \boldsymbol{Y}_{0}$ such that $C / A_{\gamma-1}$ is pure in $G / A_{\gamma-1}$ and such that

$$
\left(C / A_{\gamma-1},\left\{H_{n}, A_{\gamma-1}\right\} / A_{\gamma-1}\right) /\left(\left\{H_{n}, A_{\gamma-1}\right\} / A_{\gamma-1}\right)
$$

is pure in $\left(G / A_{\gamma-1}\right) /\left(\left\{H_{n}, A_{\gamma-1}\right\} / A_{\gamma-1}\right)$ for each $n<\omega$. Due to the purity of $A_{\gamma-1}$ and $\left\{H_{n}, A_{\gamma-1}\right\}$, we conclude that $\left\{C, H_{n}\right\}=\left\{C, H_{n}, A_{\gamma-1}\right\}$ is a pure subgroup of $G$. Obviously, there is a countable extension $J(n)$ of the subset $I(n, \gamma-1)$ such that $C \cap H_{n} \subseteq \sum_{i \in J(n)}\left(g_{i}\right)$. It follows from this if we replace $B$ by $C_{i}$ that there is an ascending sequence

$$
C_{0} \subseteq C_{1} \subseteq C_{2} \subseteq \cdots \sqsubseteq C_{k} \subseteq \cdots
$$

of pure subgroups of $G$ such that $C_{k}$ is countable over $A_{\gamma-1}$ and such that $\left\{C_{k}, H_{n}\right\}$ is pure in $G$ for all $k, n<\omega$. Letting

$$
C_{k} \cap H_{n} \subseteq \sum_{i \in j(n, k)}\left\{g_{i}\right\}
$$

where $J(n, k)$ is a countable extension of the subset $I(n, \gamma-1)$ of $I(n)$, we choose $C_{k+1} \supseteqq \sum_{i \in J(k, n)}\left\{g_{i}\right\}$ for all $n$. Define $A_{\gamma}=\bigcup_{k<\omega} C_{k}$ and observe that if we set $I(n, \gamma)=\bigcup_{k<\omega} J(n, k)$, then

$$
A_{\gamma} \cap H_{n}=\sum_{i \in I(n, r)}\left\{g_{i}\right\} \text {. }
$$

Thus conditions $(0)-(6)$ remain valid for $\alpha<\gamma$. We have ignored condition (7) in the selection of $A_{\gamma}$, but there is no problem in choosing the $A_{\alpha}$ 's such that they exhaust $G$. For example, if the index set $I(n)$ is chosen to be the set of ordinals less than $\boldsymbol{\aleph}_{n}$, then we can easily carry along the condition that $\alpha \in I(n, \alpha)$ for all $n<\omega$ provided that $\alpha \in I(n)$.

In order to show that $G$ is a direct sum of cyclic groups it remains only to show that $A_{\alpha}$ is a direct summand of $A_{\alpha+1}$ for each $\alpha<\mu$. Since $A_{\alpha}$ is pure and since $A_{\alpha+1} / A_{\alpha}$ is countable, it is enough to show that $A_{\alpha+1} / A_{\alpha}$ is without elements of infinite height, for it is well known (and quite easy to prove) that a countable primary group without elements of infinite height is pure-projective. Suppose that $x+A_{\alpha} \in p^{\omega}\left(A_{\alpha+1} / A_{\alpha}\right) \subseteq p^{\omega}\left(G / A_{\alpha}\right)$. Then $x+A_{\alpha} \in p^{\omega}\left(\left\{A_{\alpha}, H_{n}\right\} / A_{\alpha}\right)$ for any $n$ such that $x \in H_{n}$ because $\left\{A_{\alpha}, H_{n}\right\}$ is pure in $G$. However, $p^{\omega}\left(\left\{A_{\alpha}, H_{n}\right\} / A_{\alpha}\right)=0$ since $\left\{A_{\alpha}, H_{n}\right\} / A_{\alpha} \cong H_{n} /\left(A_{\alpha} \cap H_{n}\right)$ is a direct sum of cyclic groups. Letting $A_{\alpha+1}=A_{\alpha}+C_{\alpha}$, we have that $G=\sum_{\alpha<\mu} C_{\alpha}$, 
and the theorem is proved.

The proof of the above theorem is obviously valid for the following more general result.

THEOREM 2. Suppose that the ordinal $\alpha$ is the least upper bound of a (countable) sequence of smaller ordinals. Let the primary group $G$ be a group of cardinality $\boldsymbol{\aleph}_{\alpha}$. If each subgroup of $G$ having cardinality less than $\boldsymbol{\aleph}_{\alpha}$ is a direct sum of cyclic groups, then $G$ is a direct sum of cyclic groups.

A quick review of the proof of Theorem 1 also reveals the following result.

THEOREM 3. If the primary group $G$ is the union of a countable chain of pure subgroups, then $G$ is a direct sum of cyclic groups if the subgroups are.

The following corollary is well known in case the basic subgroups are countable but not (to our knowledge) in general.

COROLlaRY 1. The union of a countable chain of basic subgroups of a primary group $G$ is again a basic subgroup of $G$.

We can glean from the proof of Theorem 1 yet a little more; the results were actually discovered the way they are presented here.

THEOREM 4. If the primary group $G$ is the set-theoretic union of a countable number of pure subgroups $G_{n}$, then $G$ is a direct sum of cyclic groups if $G_{n}$ is for each $n$.

In the proof of the above theorems we have used Prüfer's theorem that a countable primary group without elements of infinite height is a direct sum of cyclic groups, but essentially nothing beyond that is required. As an application of Theorem 4, we give a new and very simple proof of Kulikov's subgroup theorem.

COROLlaRY 2 (Kulikov). If the primary group $G$ is a direct sum of cyclic groups, then any subgroup of $G$ is a direct sum of cyclic groups.

Proof. Suppose that $G$ is a direct sum of cyclic groups and let $H$ be a subgroup of $G$. Let $G_{n}$ be an ascending sequence of pure subgroups of $G$ whose union is $G$ where $G_{n}$ is bounded by $p^{n}$. Set $A_{n}=H \cap G_{n}$ for each $n$. Let $B_{n} \supseteqq A_{n}$ be maximal in $H$ with respect 
to $B_{n} \cap p^{n} H=0$. It is a simple exercise to show that $B_{n}$ is pure in $H$. Further, $B_{n}$ is a direct sum of cyclic groups since it is bounded. Since $B_{n} \supseteqq A_{n}$, it follows immediately that $H$ is the set theoretic union of its subgroups $B_{n}$. Applying Theorem 4, we have that $H$ is a direct sum of cyclic groups.

\section{REFERENCES}

1. P. Griffith, $A$ solution to the splitting mixed group problem of Baer, Trans. Amer. Math. Soc., 139 (1969), 261-269.

2. P. Hill, Sums of countable primary groups, Proc. Amer. Math. Soc., 17 (1966), 1469-1470.

3. - The purification of subgroups of abelian groups, Duke Math. J., 38 (1970), 523-527.

4. P. Hill and C. Megibben, Extending automorphisms and lifting decompositions in abelian groups, Math. Ann., 175 (1968), 159-168.

5. - On direct sums of countable groups and generalizations, Studies on Abelian Groups, pp. 183-206, Springer-Verlag, 1968.

6. C. Megibben, The generalized Kulikov Criterion, Canadian J. Math. 21 (1969), 1192-1205.

7. R. Nunke, On the structure of Tor, II, Pacific J. Math., 22 (1967), 453-464.

8. H. Prüfer, Unendliche abelsche Gruppen von Elementen endlicher Ordnung, Dissertation, Berlin, 1921.

Received February 17, 1970 and in revised form December 14, 1971. This research was supported by NSF grant GP-12318.

Florida STATE UNIVERSity 



\section{PACIFIC JOURNAL OF MATHEMATICS}

\section{EDITORS}

H. SAMELSON

Stanford University

Stanford, California 94305

C. R. HobBY

University of Washington

Seattle, Washington 98105
J. DUGUNDJI

Department of Mathematics

University of Southern California

Los Angeles, California 90007

RICHARD ARENS

University of California

Los Angeles, California 90024

\section{ASSOCIATE EDITORS}

E. F. BECKENBACH

B. H. NeumanN

F WoLF

K. YoshidA

\section{SUPPORTING INSTITUTIONS}

UNIVERSITY OF BRITISH COLUMBIA

UNIVERSITY OF SOUTHERN CALIFORNIA

CALIFORNIA INSTITUTE OF TECHNOLOGY

UNIVERSITY OF CALIFORNIA

MONTANA STATE UNIVERSITY

STANFORD UNIVERSITY

UNIVERSITY OF NEVADA

NEW MEXICO STATE UNIVERSITY

OREGON STATE UNIVERSITY

UNIVERSITY OF OREGON

OSAKA UNIVERSITY

UNIVERSITY OF TOKYO

UNIVERSITY OF UTAH

WASHINGTON STATE UNIVERSITY

UNIVERSITY OF WASHINGTON

$\stackrel{*}{*} \stackrel{*}{*} \stackrel{*}{*}{ }^{*}{ }^{*}$ MMEICAN MATHEMATICAL SOCIETY

NAVAL WEAPONS CENTER

Printed in Japan by International Academic Printing Co., Ltd., Tokyo, Japan 


\section{Pacific Journal of Mathematics}

\section{Vol. 42, No. $1 \quad$ January, 1972}

Tage Bai Andersen, On Banach space valued extensions from split faces ........

David Marion Arnold, A duality for quotient divisible abelian groups of finite

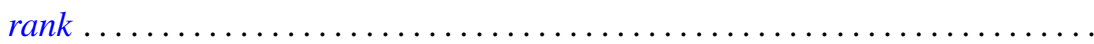

Donald Pollard Ballou, Shock sets for first order nonlinear hyperbolic

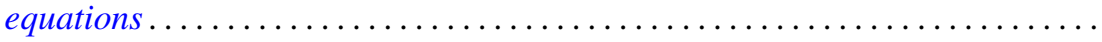

Leon Brown and Lowell J. Hansen, On the range sets of $H^{p}$ functions .........

Alexander Munro Davie and Arne Stray, Interpolation sets for analytic

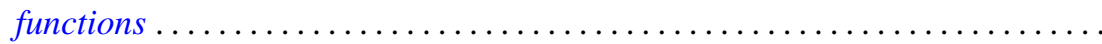

M. G. Deshpande, Structure of right subdirectly irreducible rings. II . . . . . . . . .

Barry J. Gardner, Some closure properties for torsion classes of abelian

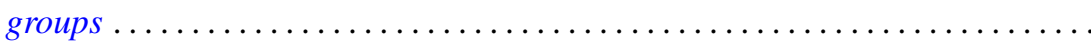

Paul Daniel Hill, Primary groups whose subgroups of smaller cardinality are

direct sums of cyclic groups . . . . . . . . . . . . . . . . . . .

Richard Allan Holzsager, When certain natural maps are equivalences .........

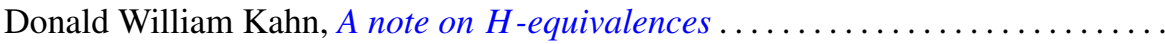

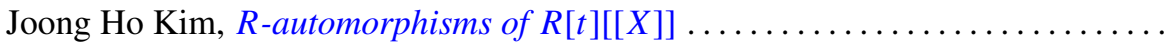

Shin'ichi Kinoshita, On elementary ideals of polyhedra in the 3-sphere.........

Andrew T. Kitchen, Watts cohomology and separability...

Vadim Komkov, A technique for the detection of oscillation of second order

ordinary differential equations .

Charles Philip Lanski and Susan Montgomery, Lie structure of prime rings of characteristic 2

Andrew Lenard, Some remarks on large Toeplitz determinants . .

Kathleen B. Levitz, A characterization of general Z.P.I.-rings. II .

Donald A. Lutz, On the reduction of rank of linear differential systems

David G. Mead, Determinantal ideals, identities, and the Wronskian ...

Arunava Mukherjea, A remark on Tonelli's theorem on integration in product

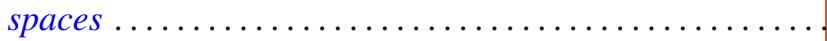

Hyo Chul Myung, A generalization of the prime radical in nonassociative rings.

John Piepenbrink, Rellich densities and an application to unconditionally nonoscillatory elliptic equations.

Michael J. Powers, Lefschetz fixed point theorems for a new class of multi-valued maps .

Aribindi Satyanarayan Rao, On the absolute matrix summability of a Fourier

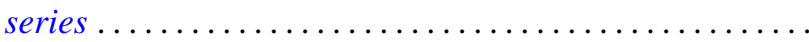

T. S. Ravisankar, On Malcev algebras ......................... 227

William Henry Ruckle, Topologies on sequences spaces . . . . . . . . . . . . . 235

Robert C. Shock, Polynomial rings over finite dimensional rings . . . . . . . . . 251

Richard Tangeman, Strong heredity in radical classes . . . . . . . . . . . . . . 259

B. R. Wenner, Finite-dimensional properties of infinite-dimensional spaces . . . . 267 